\title{
Inferring Semantic Facets of a Music Folksonomy with Wikipedia
}

\author{
Mohamed Sordo \\ Universitat Pompeu Fabra - Music Technology Group - Barcelona \\ mohamed. sordo@upf . edu \\ Fabien Gouyon \\ INESC TEC - Porto \\ fgouyon@inescporto.pt \\ Luís Sarmento \\ Amazon - Seattle, WA \\ luis.sarmento@gmail.com \\ Òscar Celma \\ Gracenote - Emeryville, CA \\ ocelma@gmail.com \\ Xavier Serra \\ Universitat Pompeu Fabra - Music Technology Group - Barcelona \\ xavier.serra@upf .edu
}

\begin{abstract}
Music folksonomies include both general and detailed descriptions of music, and are usually continuously updated. These are significant advantages over music taxonomies, which tend to be incomplete and inconsistent. However, music folksonomies have an inherent loose and open semantics, which hampers their use in many applications, such as structured music browsing and recommendation. In this paper, we present a system that can (1) automatically obtain a set of semantic facets underlying the folksonomy of the social music website Last.fm, and (2) categorize Last.fm tags with respect to the obtained facets. The semantic facets are anchored upon the structure of Wikipedia, a dynamic repository of universal knowledge.
\end{abstract}

\section{Introduction}

Music is a complex phenomenon that can be described according to multiple facets. Descriptive facets of music are commonly defined by experts (e.g. stakeholders in the music industry) in professional taxonomies, which typically include all dimensions that can be accounted for in the production and edition of a music piece (e.g. name of artists, recording studio, producer, music genre, etc.). Multifaceted descriptions are especially useful for music browsing and recommendation, as they facilitate non-linear exploration. For instance, recommendations of the Pandora 1 Internet radio use many music attributes

\footnotetext{
${ }^{1}$ http://www pandora.com/
} 
that can be grouped into facets ${ }^{2}$ such as Roots (e.g. "Afro-Latin Roots"), Instrumentation (e.g. "Mixed Acoustic and Electric Instrumentation"), Recording techniques (e.g. "Vinyl Ambience"), or Influences (e.g. "Brazilian Influences").

However, there exists no consensual taxonomy for music. Previous research showed the music industry uses inconsistent taxonomies (Pachet and Cazaly, 2000), even when restricting to a single and widespread facet such as the music genre. Also, expert-defined taxonomies (music-related or not) have two fundamental problems. First, they are very likely to be incomplete, since it is impossible for a small group of experts to incorporate in a single structure all the knowledge that is relevant to a specific domain. Second, since domains are constantly evolving, taxonomies tend to become quickly outdated in music, new genres and techniques are constantly emerging. Current lack of consensus on which are the relevant semantic facets of music, and the inherent inconsistency of some facets (e.g. genre) make the design of a consensual, complete and stable music ontology (including hundreds of facets) a daunting task.

An alternative strategy for describing music consists in relying on the broadness of the web and making use of the "wisdom of the crowds". Many music websites allow users themselves to assign their own descriptive tags to music items (artists, albums, songs, playlists, etc.). For instance, users of the social music website Last.fm ${ }^{3}$ tagged the artist Elton John as "70s", "80s", "pop", "classic-rock", "singer-songwriter", and "british", among others. Their combination of annotations provided by thousands of music users leads to the emergence of a large body of domain-specific knowledge, usually called folksonomy. Due to its informal syntax (i.e. direct assignment of tags), the tagging process allows the collective creation of very rich tag descriptions of individual music items.

When compared to taxonomies defined by experts, music folksonomies have several advantages. First, completeness, they ideally encompass all possible "ways to talk about music", including both lay and expert points of view. Second, due to the continuous nature of the tagging process, folksonomies tend to be well updated. Third, they usually incorporate both commonly accepted and generic concepts, as well as very specific and local ones. It seems reasonable to assume that folksonomies tend to encompass various groups of tags that should reflect the underlying semantic facets of the domain including not only traditional dimensions (e.g. instrumentation), but also more subjective ones (e.g. mood). For instance, figure 1 shows a picture of an artist together with a selection of tags attributed by the users of Last.fm (the complete list of tags is available on http://www.last.fm/music/Elton+John/+tags). A manual categorization of tags in figure 11 in music facets shows the richness of the tag description of that particular artist: Genre (pop, rock, classic rock), Locale (british), Time period (70s, 80s), Vocal characteristics (male vocalists), Artist characteristics (singer-songwriter) and Instrument (piano).

However, the simplicity and user-friendliness of community-based tagging imposes a

${ }^{2}$ The precise list of music attributes being a trade secret of the company. A now defunct Wikipedia page (Westergren, 2010) did provide until December 2012 a selection of such attributes.

${ }^{3}$ http://www.last.fm/ 


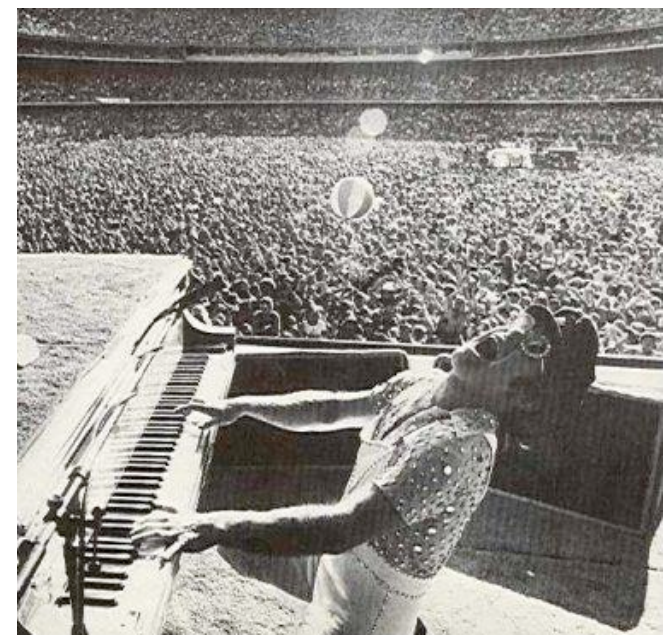

Tags:

pop

classic rock

singer-songwriter

rock

piano

british

$80 \mathrm{~s}$

$70 \mathrm{~s}$

elton john

male vocalists

Figure 1: List of Last.fm tags assigned to Elton John artist.

toll: there is usually no way to explicitly relate tags with the corresponding music facets. When browsing the tag description of a particular artist, Last.fm users browse a -albeit very rich - flat list of terms. It is for example not explicit in figure 1 that those tags related to music genre are in fact about music genre.

In this paper, we approach an essential research question that is relevant to bridging this gap: Is it possible to automatically infer the semantic facets inherent to a given music folksonomy? A related research question is whether it is then possible to classify instances of that music folksonomy with respect to the inferred semantic facets.

In this paper, we focus on the music folksonomy obtained from the social music website Last.fm. We propose an automatic method for (1) uncovering the set of semantic facets implicit to the tags of this music folksonomy, and (2) classify tags with respect to these facets. We anchor semantic facets on metadata of the semi-structured repository of general knowledge Wikipedia. Our rationale is that as it is dynamically maintained by a large community, Wikipedia should contain grounded and updated information about relevant facets of music, in practice.

This paper is structured as follows: After a review of related work, we explain our approach to obtaining the inherent semantic facets of Last.fm tags, and to automatically assigning facets to tags. Results and evaluations are proposed, we then conclude with a summary and directions for future work.

\section{Related work}

\subsection{Tag categorization}

Bischoff et al. (2009) propose an approach, using rule- and model-based methods, to automatically infer the semantic category (facet) of the tags. Rule-based methods rely on regular expressions and predefined lists, whilst the model-based ones employ attributes 
such as: tag popularity, number of words, number of characters, part of speech, and word sense disambiguation (using WordNet ${ }^{4}$ ). The list of facets used is: Topic, Time, Location, Type, Author/Owner, Opinions/Qualities, Usage, and Self reference. Then, they compare automatic tag classification against a ground truth of around 2,100 manually classified tags based on these list of facets. Experimental results using Last.fm, Delicious.com and Flickr datasets show that these two methods can identify tag facets with an accuracy higher than $80 \%$. The results vary significantly across the different domains. Nonetheless, the manual creation of the ground truth-using a handcrafted lists of terms - limits the coverage for these three different domains.

Except the work presented by Bischoff et al. (2009) that includes the music domain in their evaluations, most of the previous work has been focused on the image domain. Overell et al. (2009) present a method for classifying Wikipedia articles using structural patterns as features, and WordNet semantic categories as a classification scheme. Then, they apply this method to also classify Flickr tags to WordNet semantic categories. Their results show an increase by $115 \%$ of the Flickr vocabulary coverage, compared to the WordNet baseline. A similar approach by the same authors is also presented in (Sigurbjörnsson and van Zwol, 2008).

\subsection{Social Tagging in Music}

Music tags have recently been the object of increasing attention by the research community (Lamere, 2008; Celma, 2010). A number of approaches have been proposed to associate tags to music items (e.g. a particular artist, or a music piece) based on an analysis of audio data (Bertin-Mahieux et al., 2008; Turnbull et al., 2008), on the knowledge about tag co-occurence (Levy and Sandler, 2008), or on the extraction of tag information from community-edited resources (Sarmento et al., 2009). However, in most cases, such approaches consider tags independently, i.e. not as instances in structured hierarchies of different music facets. When hierarchies of facets are considered, they are usually defined a priori, and greatly vary according to authors. For example, Lamere (2008) groups tags in the following facets: Genre, Locale, Mood, Opinion, Instrumentation, Style, Time period, Recording label, Organizational, and Social signaling.

Alternatively, Pachet and Roy (2009) use 935 labels grouped in 16 facets: Style, Genre, Musical setup, Main instruments, Variant, Dynamics, Tempo, Era/epoch, Metric, Country, Situation, Mood, Character, Language, Rhythm and Popularity. Aucouturier (2009) considers 801 labels grouped in 18 facets, the same as (Pachet and Roy, 2009), with the exception of Popularity, and 3 extra facets: Affiliate, Special creative period, and Text category. On the other hand, Turnbull et al. (2008) use 135 concepts grouped in only 6 facets: Instruments, Vocal characteristics, Genres, Emotions, Acoustic quality and Usage.

To our knowledge, however, few efforts have been dedicated to the particular task of automatically identifying the relevant facets of music tags. In their work on inferring models for genre and artist classification, Levy et al. apply dimensionality reduction

\footnotetext{
${ }^{4}$ http://wordnet.princeton.edu/
} 
techniques to a data set of tagged music tracks in order to obtain their corresponding compact representations in a low-dimensional space (Levy and Sandler, 2008). They base their approach on tag co-occurrence information. Some emerging dimensions can be associated to facets such as Era (e.g. the dimension [90s]). However, most of the dimensions thus inferred are, in fact, a combination of diverse music facets, such as for example the dimension [guitar; rock], which includes concepts of Instrumentation and of Genre, or [seen live; world music] (including concepts of Social signaling and Genre), or [new wave; 80s] (including concepts of Genre and Time period).

Cano et al. (2004) use the WordNet ontology to automatically describe sound effects. Albeit the very large amount of concepts in WordNet, they report that it accounts for relatively few concepts related to sound and music, and propose an extension specific to the domain of sound effects. On the one hand, they illustrate that browsing can indeed be greatly enhanced by providing multifaceted descriptions of items. On the other hand however, it is our belief that, because of their necessary stability, existing ontologies are not the most adapted tool to describe domains of knowledge with inherent open and dynamic semantics, such as music.

\section{$2.3 \quad$ Expert-defined music facets}

We reviewed the music facets most referred to in the literature, which resulted in the selection of 45 music facets, detailed in table 1. In this review, we refrained from attempting to group together facets with apparent polysemic meanings (e.g. Style, Rhythm) or facets with different denominations yet apparent similar meanings (e.g. Style and Stylings, or Acoustic qualities and Texture). From the list of referenced literature, a special mention should be made to the Music Ontology (Raimond et al., 2007, 2010 ), which is an attempt to provide a vocabulary for linking a wide range of musicrelated information. The ontology is a formal framework for dealing with music-related information on the Semantic Web, including editorial, cultural and acoustic information.

Obtaining detailed taxonomies for all these facets is an elusive task because of two factors: the current lack of consensus in musical taxonomies, and the scarcity of resources (for instance, most of the 45 expert-defined facets do not provide the full list of instances for each facet used nor the full mapping between facets and their instances). Nevertheless, we gathered specific expert taxonomies for four particular facets, chosen for their relevance in current literature, namely, Genre, Mood, Musical Instruments and Country and Language. See section 4.1.1 for more details. 


\begin{tabular}{|c|c|c|}
\hline Facet & Example (as used in the literature) & References (non exhaustive list) \\
\hline Genre & Blues & Raimond et al. 2010) \\
\hline Style (1) & BeBop & $\begin{array}{l}\text { (Pachet and Roy 2009 Aucou- } \\
\text { turier 2009) }\end{array}$ \\
\hline Style (2) & Political, Humor & $\begin{array}{l}\text { (Lamere 2008 } \\
\text { et al. } 2008)\end{array}$ \\
\hline Leanings/Stylings & Classical Stylings & (Westergren, 2010) \\
\hline Character & Child-oriented & $\begin{array}{l}\text { Pachet and Roy 2009) Aucou- } \\
\text { turier 2009) }\end{array}$ \\
\hline Roots & Acid jazz roots, Funk roots & Westergren 2010 \\
\hline Influences & Flamenco influences & (Westergren, 2010) \\
\hline Time period, era/epoch & 70s, 1989, Romantic period & Levy and Sandler 2008) \\
\hline Recording label & Kill Rock Stars & Lamere 2008) \\
\hline Locale, country, nationality & Germany & $\begin{array}{l}\text { Lamere } 2008 \text { Bertin-Mahieux } \\
\text { et al. } 2008)\end{array}$ \\
\hline Language & Spanish & $\begin{array}{l}\text { (Pachet and Roy 2009) Aucou- } \\
\text { turier 2009) }\end{array}$ \\
\hline Musical setup & String ensemble & $\begin{array}{l}\text { (Pachet and Roy 2009) Aucou- } \\
\text { turier 2009) }\end{array}$ \\
\hline Main instrument & Double-bass & $\begin{array}{l}\text { (Pachet and Roy 2009) Aucou- } \\
\text { turier 2009) }\end{array}$ \\
\hline Instrumentation, instrument & Piano, female vocal & $\begin{array}{l}\text { (Duan et al. 2008 Westergren } \\
2010)\end{array}$ \\
\hline Instrumentation & Acoustic rock instrumentation & (Westergren, 2010) \\
\hline Orchestration, arrangement & $\mathrm{n} / \mathrm{a}$ & Raimond et al. 2010 \\
\hline Performance & $\mathrm{n} / \mathrm{a}$ & (Raimond et al. 2010 \\
\hline Vocal characteristics & Agressive, breathy & (Turnbull et al. 2008) \\
\hline Vocals (1) & Male, group & (Duan et al. 2008) \\
\hline Vocals (2) & $\begin{array}{l}\text { Breathy, unintelligible vocal deliv- } \\
\text { ery }\end{array}$ & Westergren 2010 \\
\hline Acoustic qualities & $\begin{array}{l}\text { Catchy, heavy beat, fast tempo, } \\
\text { acoustic texture }\end{array}$ & (Turnbull et al. 2008) \\
\hline Variant & Natural, acoustic & $\begin{array}{l}\text { Pachet and Roy 2009 } \\
\text { turier 2009) }\end{array}$ \\
\hline Texture & Acoustic & Duan et al. 2008) \\
\hline Production & Studio, live & (Duan et al. 2008 \\
\hline Recording techniques & Vinyl ambience, studio production & (Westergren 2010 \\
\hline Musical qualities & Easy listening qualities & (Westergren, 2010) \\
\hline Dynamics & Decreasing & $\begin{array}{l}\text { (Pachet and Roy 2009) Aucou- } \\
\text { turier 2009) }\end{array}$ \\
\hline Tempo & Slow, $120 \mathrm{BPM}$ & $\begin{array}{l}\text { (Pachet and Roy, 2009 Duan } \\
\text { et al. 2008) }\end{array}$ \\
\hline Metric & $3 / 4,4 / 4$ & $\begin{array}{l}\text { (Pachet and Roy 2009 Aucou- } \\
\text { turier 2009) }\end{array}$ \\
\hline Rhythm (1) & Groovy & $\begin{array}{l}\text { (Pachet and Roy 2009 Aucou- } \\
\text { turier 2009) }\end{array}$ \\
\hline Rhythm (2) & Strong & Duan et al. 2008 \\
\hline Feel & Driving shuffle feel & (Westergren, 2010) \\
\hline Tonality & Major, minor & $\begin{array}{l}\text { Duan et al. 2008 Westergren } \\
2010)\end{array}$ \\
\hline Structures & Basic Rock Song Structures & Westergren, 2010) \\
\hline Organizational, situation & City by night, must check out & $\begin{array}{l}\text { (Pachet and Roy 2009 Aucou- } \\
\text { turier 2009) }\end{array}$ \\
\hline Usage & Waking up, music for making love & (Turnbull et al. 2008) \\
\hline Social signaling & Seen live & (Lamere 2008) \\
\hline Mood, emotion & Agressive, happy & $\begin{array}{l}\text { (Lamere 2008) Laurier et al. } \\
2009)\end{array}$ \\
\hline Affective & Positive, neutral, negative & Duan et al. 2008) \\
\hline Arousal & Strong, middle, weak & (Duan et al., 2008) \\
\hline Opinion, preference & Love, favourite & $\begin{array}{l}\text { Lamere 2008) Levy and Sandler } \\
2008)\end{array}$ \\
\hline Popularity & High, medium, low & (Pachet and Roy, 2009) \\
\hline Lyrics & $\mathrm{n} / \mathrm{a}$ & Raimond et al. 2010$)$ \\
\hline Lyrical content & $\begin{array}{l}\text { Political lyrics, cash-obsessed } \\
\text { lyrics }\end{array}$ & (Westergren 2010$)$ \\
\hline Place, festival & Glastonbury & (Raimond et al. 2010) \\
\hline
\end{tabular}

Table 1: Expert-defined music facets and corresponding examples of use in the literature and references. Different meanings of a facet appear in different lines. 


\section{Method}

Our method consists in using metadata from Wikipedia to infer the semantic facets of the Last.fm music folksonomy. This is performed in two steps. In the first step, we specialize the very large network of interlinked Wikipedia pages to the specific domain of the Last.fm music folksonomy. This is done by maximizing the overlap between Wikipedia pages and a list of frequent tags from the folksonomy. As the resulting network still represents a very large number of nodes, in a second step, we focus on the most relevant ones (node relevance being defined as an intrinsic property of the network). This step also includes additional refinements.

\subsection{Data}

Our data consists of a large dataset of artist tags gathered from Last.fm during April 2010 via the API provided by Last.fm.5. The dataset consists of around 600,000 artists and 416,159 distinct tags. Tags were retrieved using the API method artist.getTop Tags $5^{6}$, Each tag includes a relevance weight 7 which reflects the relevance of the tag to the queried artist.

This dataset was cleaned in order to remove noisy/irrelevant data: (1) tags were edited in order to remove special characters such as spaces, etc.; (2) tags were filtered by weight, only tags with a weight $\geq 1$ were kept; and (3) tags were filtered by usage, keeping only those tags that were applied $\geq 10$ times. As a result, the final dataset consists of 582,502 artists, 39,953 distinct tags, and an average of 9 tags per artist.

A special note should be made here regarding the fact that we are not using tags applied to individual music tracks but to artists. One might think that describing e.g. Tonality, or Rhythm, might be more accurate at the level of tracks rather than artists. However, we noticed a greater tendency for noise in tracks' tags in comparison to artists' and decided to use the latter kind. Applying more sophisticated Natural Language Processing techniques than what is described in this paper might permit to effectively clean tracks' tag data and potentially result in more reliable data for further experiments. This is left for future work.

\subsection{Obtaining a music-related network}

Wikipedia pages are usually interlinked, and we use the links between two particular types of pages (i.e. articles and categories) to construct a music-related network. Concretely, we use the DBpedia ${ }^{8}$ knowledge base, that provides structured, machinereadable descriptions of the links between Wikipedia pages (DBpedia uses the SKOS vocabulary, in its 2005 version) $!^{9}$ In particular, we make use of two properties that

\footnotetext{
${ }^{5}$ http://www.last.fm/api

${ }^{6}$ http://www. last.fm/api/show/artist.getTopTags

${ }^{7}$ i.e. Last.fm "count" or "relevance weight", which goes from 0 to 100

${ }^{8}$ http://dbpedia.org/

${ }^{9}$ http://www .w3.org/TR/2005/WD-swbp-skos-core-spec-20051102/
} 
connect pages in DBpedia: (1) the property subjectOf, which connects articles to categories (e.g. the article "Samba" is a subjectOf of the category Dance_music, and (2), the property broader $O f$, which connects categories in a hierarchical manner (e.g. the category Dance is a broaderOf of the category Dance_music, which is a broaderOf of the category Ballroom_dance_music).

We start from the seed category Music and explore its neighbourhood from the top down, checking whether connected categories can be considered relevant to the music domain. A category is considered relevant if it satisfies any of the two following conditions:

- It is a Last.fm tag, such as for example "Rock and Roll". (This condition will be referred to as isMusical);

- At least one of its descendants is a tag from Last.fm and the substring "music" is included in the title or the abstract of the corresponding Wikipedia article. (This condition is further referred to as isTextMusical.)

The descendants of a category are fetched from DBpedia using the two connecting properties previously described. These descendants can be either "successors" (i.e. all direct subjectOf and broaderOf of this category), or successors of successors, and so on. This iterative search is limited by a maximum depth, empirically fixed to a value of 4. Indeed, experiments with smaller values yielded a significant reduction of the tag coverage, while experiments with greater values did not increase significantly the coverage.

If any of the previous conditions is satisfied, the category, its successors and their edges are added to the network. Otherwise, the category and all incident edges are removed. The algorithm proceeds iteratively (following a Breadth-First search approach) until no more categories can be visited. A summarized version of the method for obtaining a music-related network is described in Algorithm 1 .

It is important to note that the Wikipedia categorization (and thus its DBpedia representation) is prone to human errors. For instance, despite the Wikipedia editorial recommendations ${ }^{10}$ it can happen that a given category (e.g. New_Weird_America) would be both a subcategory and a super-category of the same category (e.g. Freak_folk), resulting in a cycle (in graph theory), that is, an infinite loop. We tackle this issue by keeping track of the nodes that are already added to the network, and not including links that could form a cycle in the network.

\subsection{Finding relevant facets}

Once the network of music-related facets is built, the next step is to find the nodes that are potentially more relevant to the network than others.

We invert the direction of the edges of the network in order to point back in the direction of the most generic category, i.e. Music, and we compute the PageRank of

\footnotetext{
${ }^{10}$ http://en.wikipedia.org/wiki/Wikipedia:SUBCAT
} 


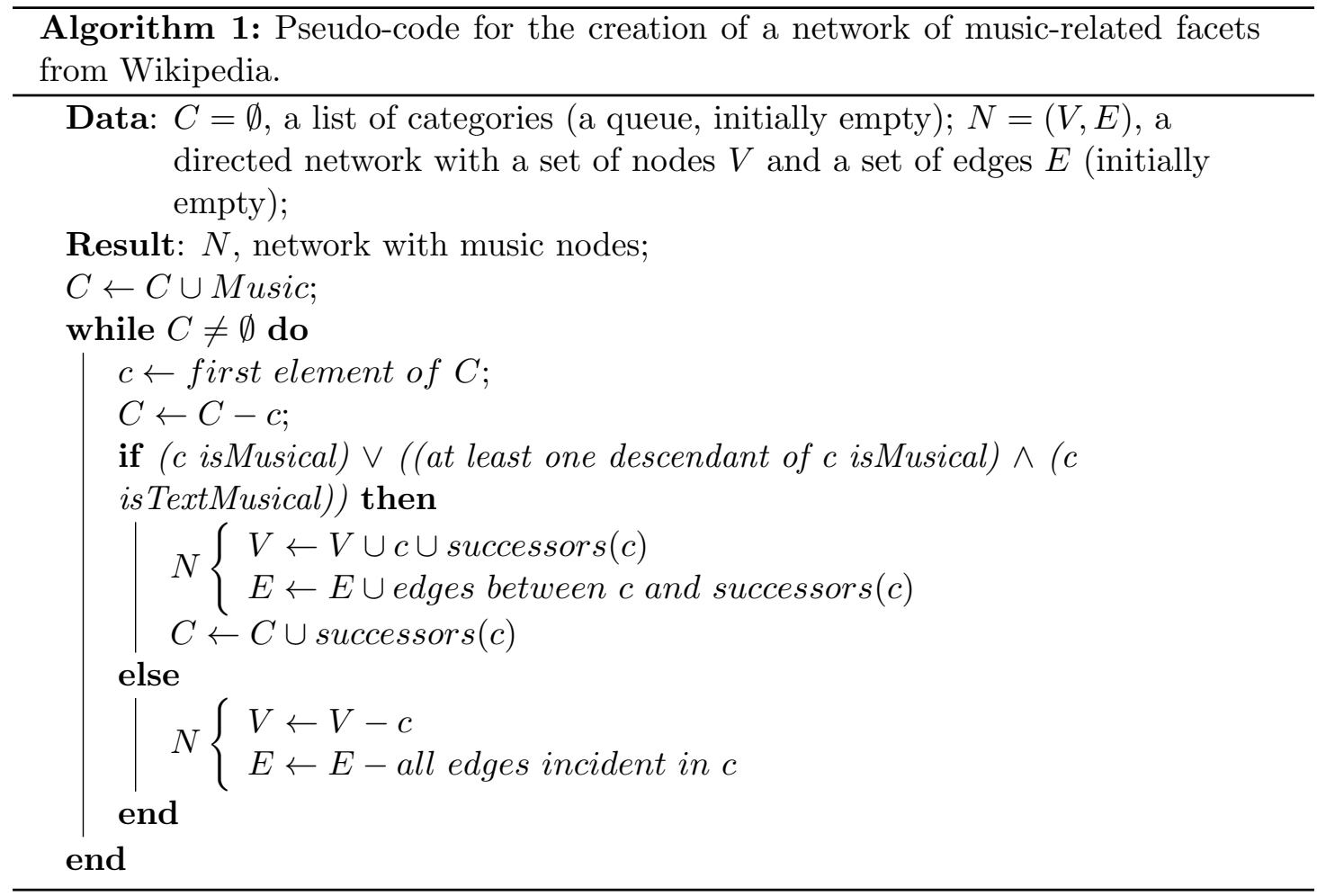

the resulting network. PageRank (Page et al. 1999$)$ is a link analysis algorithm that measures the relative relevance of all nodes in a network. In PageRank, each node is able to issue a relevance vote on all nodes to which it points to (thus the need for reorienting the edges). The weight of the vote depends on the relevance of the voting node (i.e. relevant nodes issue more authoritative votes). The process runs iteratively, and (under certain conditions) converges to a stable relative ranking, where nodes to which more edges from other relevant nodes converge (directly or indirectly) are considered more relevant. For initializing the PageRank algorithm, we set the initial weight of each node to 0 .

In order to capture general yet complementary facets of music, we aim at reducing semantic overlap as much as possible by applying the following filters:

Stub Filter: We remove all categories with substring "_by_" and "_from_". We noticed that many categories in Wikipedia are actually combinations of two more general categories, as for instance Musicians_by_genres, which is halfways between Musicians and Music_genres (see also figure 2). Further, we also remove categories that include "-music(al)_groups" (e.g. Musical_groups_from_California that has hundreds of connected categories, hence a high PageRank). Most of these categories are used as stubs, even sometimes explicitly so we also excluded categories with the word "stub". 
Over-Specialization Filter: We exclude all categories that include lexically a more relevant category. Many relevant categories are specializations of other more relevant ones, this occurs mostly with concepts related to anglophone music, which are described in great detail in Wikipedia (e.g. American_Musicians includes Musicians that has a higher PageRank).

Tag Filter: We remove all categories that are Last.fm tags. Our objective is to uncover music facets that are implicit to the tags that make up the folksonomy. In general, tags are instances of such facets, not the facets themselves.

\subsection{Assigning facets to tags}

In order to assign a set of facets to a given Last.fm tag, we process the subnetwork of Wikipedia pages specialized to the Last.fm folksonomy (obtained in section 3.2), as described in Algorithm 2 (note that this process is restricted to tags that can be matched to one of the nodes in the network).

Given a Last.fm tag $t$, we look at its predecessor categories $c$, or more formally:

$$
\text { predecessors }(t)=\{c \mid(t \in \text { broaderOf }(c)) \vee(t \in \operatorname{subject} O f(c))\}
$$

If any of these predecessors is a top- $\mathrm{N}$ facet, ordered by PageRank (we set $N=50$ ), it is then assigned to $t$. The process continues iteratively until no more facets can be assigned to the tag, or a maximum number of iteration (maxIter) is exceeded. This condition can be interpreted as the maximum distance in the network between a tag and a facet.

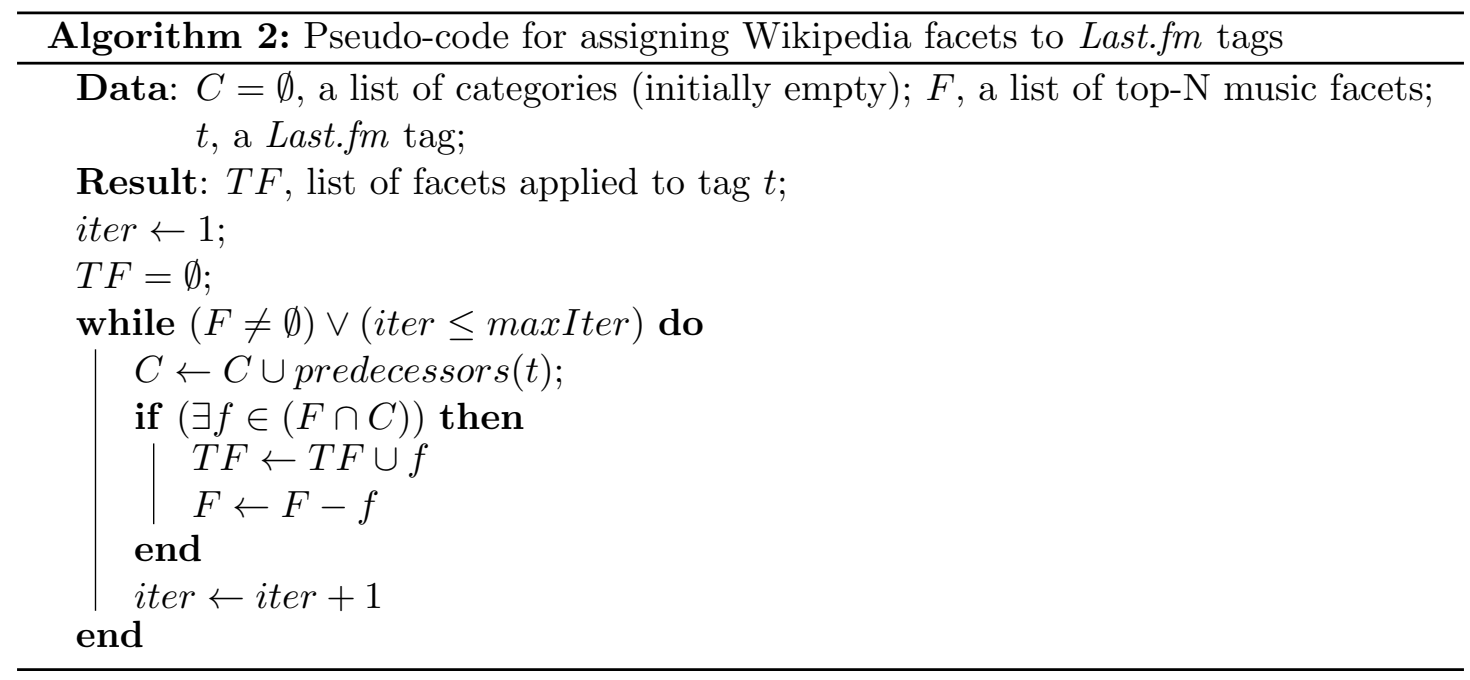




\subsection{Tag/Facet Relevance}

The relevance $R_{t f}$ of a music facet $f$ to a tag $t$ is computed as the normalized inverse distance $d_{t f}$-in number of successive edges - between $t$ and $f$ :

$$
R_{t f}=\frac{d_{t f}^{-1}}{\sum_{i} \frac{1}{d_{t i}}}
$$

An example of tag/facet relevance is provided in figure 2 .

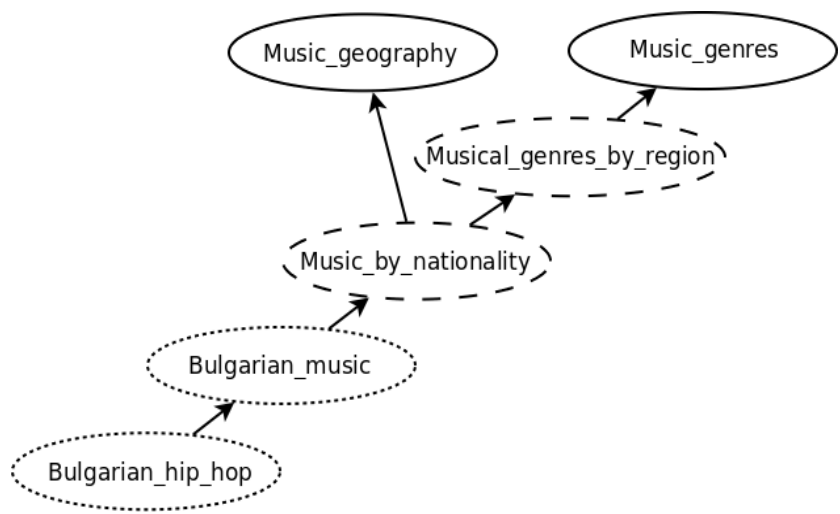

Figure 2: Example of subnetwork in our data. Dotted lines correspond to Wikipedia categories that are also Last.fm tags. Dashed lines correspond to categories not kept. Plain lines correspond to facets kept. 


\begin{tabular}{|c|c|}
\hline Music_genres & Aspects_of_music \\
Music_geography & Hip_hop_genres \\
Musical_groups & Music_of_California \\
Music_industry & Music_theory \\
Musicians & Rock_and_Roll_Hall_of_Fame_inductees \\
Musical_culture & Musical_subcultures \\
Occupations_in_music & Recorded_music \\
Music_people & Musical_quartets \\
Record_labels & Music_festivals \\
Music_technology & East_Asian_music \\
Sociological_genres_of_music & Centuries_in_music \\
Music_publishing_companies & Musical_composition \\
Musical_instruments & Musical_quintets \\
Anglophone_music & Southern_European_music \\
Music_of_United_States_subdivisions & Music_software \\
Western_European_music & Incidental_music \\
American_styles_of_music & Years_in_music \\
Radio_formats & Music_websites \\
Music_publishing & Guitars \\
Albums & Music_competitions \\
Musical_techniques & Musical_eras \\
Wiki_music & Music_and_video \\
Music_history & Musical_terminology \\
Music_performance & Music_halls_of_fame \\
Music_publishers_"people" & Dates_in_music \\
\hline & \\
\hline &
\end{tabular}

Table 2: Top-50 Wikipedia music facets

\begin{tabular}{|c|c|c|c|}
\hline Music_genres & Occupations_in_music & Musical_instruments & Aspects_of_music \\
\hline Sufi_music & Troubadour & Melodica & Rhythm \\
Dance_music & Bandleaders & Tambourine & Melody \\
Indietronica & Pianist & Drums & Harmony \\
Minimalism & Singer-songwriter & Synthesizers & Percussion \\
Singer-songwriter & Flautist & Piano & Chords \\
\hline Music_software & Music_websites & Music_competitions & Musical_eras \\
\hline Nanoloop & Mikseri.net & Nashville_Star & Baroque_music \\
Scorewriter & PureVolume & American_Idol & Ancient_music \\
MIDI & Allmusic & Melodifestivalen & Romantic_music \\
DrumCore & Jamendo & Star_Search & Medieval_music \\
Renoise & Netlabels & Eurovision_Song_Contest & Renaissance_music \\
\hline
\end{tabular}

Table 3: Random sample of tags inferred for various music facets

\section{Results and Evaluation}

After running both stages of our method, we obtained a list of 333 candidate facets and 3596 tags. One can notice here that Wikipedia articles cover slightly less than $10 \%$ of our initial Last.fm tag data. Further discussion on this aspect can be found in Section 5 .

Table 2 contains the top-50 facets, ordered by pagerank (top to bottom, left to right).

Table 3 presents a subset of the obtained facets, followed by a subset of their cor- 
responding list of top tags. Top tags are chosen based on the distance (in number of successive edges in the music network) to the given facet.

Figure 2 illustrates an example of subnetwork in our data. Given the tag "bulgarian hip-hop", our method starts navigating through the predecessors of this tag until finally reaching two music facets: Music_genres and Music_geography. In this particular example, computing the relevance of each facet with Equation 1 yields the following:

bulgarian hip-hop: \{(Music_genres, 0.4),

(Music_geography, 0.6)\}

\subsection{Evaluation methodology}

In this section, we propose to evaluate two aspects of our system. First, in section 4.2, we evaluate the quality of the facets inferred: Is our system able to infer commonly-accepted facets akin to the ones found in taxonomies designed by experts? Is our system able to effectively infer useful facets from the broad, and up-to-date, domain-specific knowledge about music contained in Wikipedia? In order to evaluate the quality of inferred facets, we make use of the expert-defined facets mentioned in section 2.3 .

Second, in section 4.3, we evaluate the classification of tags with respect to the inferred facets: Are the tag assignments done by our system similar to what could be found in expert-defined classification systems? In order to evaluate tag classification, we can also make use of the expert-defined facets mentioned in section 2.3, however for this task, we need more than just accepted facets, we also need a ground truth for tags/facets assignments.

Obtaining detailed taxonomies for the 45 expert-defined facets mentioned in section 2.3 is an elusive task. Hence we only focus on four particular facets that are especially relevant in current literature, namely, Genre, Musical Instruments, Country and Language, and Mood. We call this evaluation dataset our Gold Standard, which is detailed in section 4.1.1.

As put forward in the introduction of this paper, a central objective to our system is to be able not only to infer music facets and replicate tag/facet classification that could be found in expert systems, but more importantly to provide a richer and more up-to-date representation of "the words of music." In order to evaluate that particular aspect, we propose to focus part of the evaluation on the comparison of our system with another tag classification system which we call Baseline System (detailed in section 4.1.2). Following the evaluation methodology of (Overell et al. 2009), the Baseline System replicates the behavior of our system, but instead of relying on community-based information from Wikipedia, it relies on expert-based information from WordNet.

The two systems are first compared in section 4.3 in terms of tag coverage with respect to the Gold Standard, and also in terms of general quality of tag/facet assignments.

A last evaluation in section 4.4 aims at testing the applicability of our system and at verifying whether community-based information can produce a better faceted description of artists' tag sets than expert-based information. In this endeavour, we compare our 
system with the Baseline system in the task of identifying the facets that exist in the tag description that users have assigned to Last.fm artists.

\subsubsection{Gold Standard}

In this paper, the Gold Standard (GS) is a list of tags/facets assignments gathered from expert information sources that will serve as ground-truth for evaluation. It is restricted to four particular facets that are especially relevant in current literature on music tags.

Expert-defined instances of the four GS facets have been gathered from Allmusic (http://allmusic.com/) for the Genre facet, from references in the psychological literature and taxonomies used in MIREX evaluations (Laurier et al., 2009) for the Mood facet, from MusicBrainz (http://musicbrainz.org/) for the Musical Instruments facet and from the United Nations (http://unstats.un.org/), Index Mundi (http://www . indexmundi.com/) and Ethnologue (http://www.ethnologue.com/) for the Country and Language facet. There is a total of 1715 tags for the 4 facets in our Gold Standard. Table 4 describes the number of tags for each one of these facets, as well as some examples of these tags.

\begin{tabular}{|c|c|c|}
\hline GS Facet & Tags per facet & examples \\
\hline Genres & 711 & rock, ambient dub, tango, post-punk, etc. \\
Instruments & 280 & bass guitar, trombone, flugelhorn, lute, etc. \\
Locations \& Languages & 609 & arabic, hungarian, spain, etc. \\
Moods & 115 & excited, dark, calm, happy, etc. \\
\hline
\end{tabular}

Table 4: Number of tags per facet and example of tag/facet assignments in the Gold Standard.

\subsubsection{Baseline system}

The creation of the Baseline System follows the evaluation methodology of (Overell et al., 2009). It is based on a method for network creation and tag categorization that follows a similar overall rationale than the method described in section 3.2 (for the network creation part) and section 3.4 (for the tag categorization part), but it is built on top of expert knowledge from WordNet. Namely, the Baseline System:

1. Uses a third-party knowledge repository (WordNet).

2. Creates a music-related network, in a top-down fashion, starting with initial selected "seeds" from WordNet, and following the links inherent to the knowledge base at hand, adding descendants iteratively to the network.

3. Navigates the network from the bottom to the top, starting from tags of the Last.fm music folksonomy, pruning nodes in the network, and attributing facets to tags.

The main difference between the Baseline system and our proposed method (section 3) are: 
1. The use of WordNet instead of Wikipedia as third-party knowledge repository;

2. The connecting properties from WordNet we use are meronyms and hyponyms;

3. The Baseline system does not try to infer facets, they are defined beforehand (expert-defined facets in table 1), and serve as starting seeds to the algorithm (instead of the single Music seed in our proposed sytem).

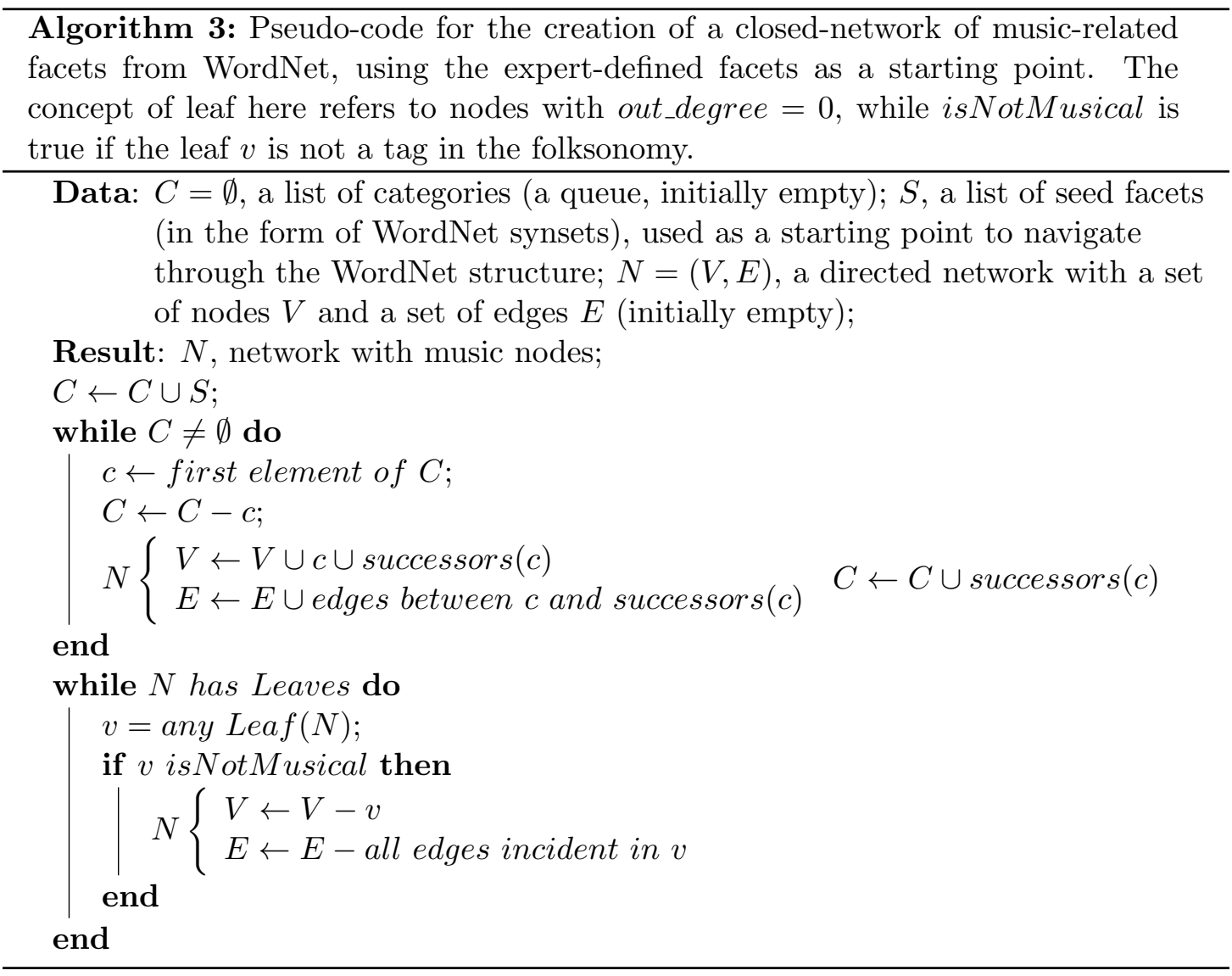

For further details, see Algorithm 3. It is important to emphasize the fact that, unlike our system, the Baseline system does not infer facets. It uses expert-defined facets as background knowledge, retrieve corresponding concepts in WordNet and uses WordNet link structure to assign tags to facets. However, not all of the 45 expert-defined facets (table 1) can be matched to WordNet concepts. Only 36 facets were matched. Expert-defined facets that could not be matched to WordNet concepts are: Acoustic qualities, Affective, Dynamics, Lyrical content, Main instrument, Musical qualities, Recording techniques, Social signaling, Variant, and Vocal characteristics. 


\subsection{Evaluating inferred facets}

Our system is able to infer 333 facets (table 2 shows the top-50 ones). As a first step in the evaluation of the inferred facets, we performed a manual match between the system's facets and expert-defined ones (see Table 1).

In a first stage, we proceed in a strict fashion, only considering matches that can be unequivocally done. This results in matching 8 inferred facets, and are presented in Table 5 .

As shown in Table 5, our system ranks relevant facets (i.e. expert-defined facets) relatively high. Indeed, seven out of eight (i.e. all except Music_Production) of the expert-defined facets that our system could infer are ranked among the 50 most relevant facets.

\begin{tabular}{|c|c|c|}
\hline Inferred facet & Expert-defined facet & Inferred facet PageRank \\
\hline Music_genres & Genre & 1 \\
Music_geography & Locale, country, nationality & 2 \\
Record_labels & Recording label & 9 \\
Musical_instruments & Instrumentation, instrument & 13 \\
Music_performance & Performance & 24 \\
Music_festivals & Place, festival & 34 \\
Musical_eras & Time period, era/epoch & 46 \\
Music_production & Production & 242 \\
\hline
\end{tabular}

Table 5: Strict match between inferred and expert-defined facets

In a second stage, we consider also approximate matches, where one can argue that inferred and expert-defined facets do correspond to similar or related concepts. For a total of 33 additional expert-defined facets, we could produce such "soft" matches. For instance, for some of the inferred facets presented in Table 5, we can also obtain the following "soft" matches: Music_geography can be matched to Language, Musical_instruments can be matched to Main instrument, or even Instrumentation. Music_production can also be matched to Recording techniques. Musical_scales can be matched to Tonality. Additionally, we can also find matches between some inferred facets and other, more specific, expert-defined-facets. For instance Aspects_of_music can be matched to Rhythm, to Tempo, to Metric, to Tonality, and to Dynamics. Conversely, some inferred facets are more specific than corresponding expert-defined facets. For example, Centuries_in_music, Years_in_music, Musical_eras, and Dates_in_music can be matched to the single expert-defined facet time period, Era/epoch.

Globally, i.e. up to rank 242, considering both "soft" and strict matches, we are able to match 41 expert-defined facets, while the Baseline system matches 36 expert-defined facets.

We could also notice cases in which our system was not able to infer correct facets for expert-defined concepts despite the fact that they correspond to existing categories in Wikipedia. For instance, Rhythm and Lyrics were not inferred because they are also Last.fm tags and our system includes a Tag Filter (see section 3.3). We propose a 
solution to this issue in the last section of this paper, related to future work.

It is important to notice here that some facets that were inferred do not correspond to any of the expert-defined facets, yet they are potentially useful for music categorization. For instance, the facet Music_publishing_companies inferred by our system (see Table 21 is clearly relevant (at the very least to actors in the music publishing business), yet is not present in our list of expert-defined facets. Another example are the inferred facets Music_competitions, or Music_websites (see Table 3). Other examples of such unusual music facets that are not typically part of music taxonomies, hence typically not used for music exploration, include e.g. Musical_subcultures, and Music_history. The following examples aim at showing how using these facets would improve faceted music browsing:

$$
\begin{gathered}
\text { Musical_subcultures } \rightarrow \text { Rockabilly } \rightarrow \text { Psychobilly } \\
\text { Musical_subcultures } \rightarrow \text { Grunge_music } \rightarrow \text { Post }- \text { grunge } \\
\text { Musical_subcultures } \rightarrow \text { Punk } \rightarrow \text { Conservative_Punk } \\
\text { Music_history } \rightarrow \text { Music_history_by_genre } \rightarrow \text { Non-Western_classical_music_genres } \rightarrow \text { Qawwali } \\
\text { Music_history } \rightarrow \text { History_of_musical_instruments } \rightarrow \text { Early_musical_instruments } \rightarrow \text { Guqin }
\end{gathered}
$$

There are also examples of inferred facets which are difficult to evaluate when comparing to expert-defined taxonomies. For example, our system infers the facet Guitars, to which are assigned Last.fm tags such as "Bass_guitars", "Portuguese_guitar", "John_Frusciante", or "The_Beatles". Clearly, there is no match with any expert-defined facet. However, one could argue that expert-defined taxonomies focus on describing the qualities of the music itself, and leave aside other parts of the universe of music, that are in fact meaningful to (at least some) users. Indeed associating The Beatles to a "guitar-type music" does make sense.

Another important aspect of our system is the specifity of the inferred tags, such as "iPhone", "American Idol", "Jamendo", "Garage Band", or even artist's names, which could not be found in the Baseline system. For instance, our system proposes the following description for the tag "iPhone":

IPhone: \{(Music_software, 0.625),

$$
\text { (Music_technology, 0.375)\} }
$$

These particular examples can show the importance of using a community-edited knowledge repository, in terms of being more up-to-date. Nevertheless, there is no way to objectively evaluate such new and unseen tags (instances in classification). This task can only be done by performing a human evaluation of these inferred tags.

Looking at the list of top facets generated by our method, we can also observe that there is a certain bias towards anglophone music. For example among the top 50 facets, there are four facets that are clearly related with Anglophone culture - Anglophone Music, Music of United States subdivisions, American styles of music, or even 
Music of California - while there are only two facets explicitly related with other parts of the world -East Asian Music and Southern European Music. We believe that this is a direct consequence of several factors. First, despite the fact that Last.fm's music repository is open to many different cultures and sub-cultures, musical content and corresponding tag assignment is probably biased towards anglophone music. Second, the Last.fm's folksonomy itself is mostly in English. Third, the english-language version of the Wikipedia used by our methods has obviously an intrinsic bias towards anglophone cultures.

\subsection{Evaluating assignment of facets to tags}

Another important aspect that we want to evaluate is the assignment of (inferred) facets to tags: we wish to evaluate whether the different facets we infer are correctly related with tags. For that we focus on the four facets that are part of our Gold Standard (see table (4). For each of these four reference facets, we compare the tag/facet assignments of our system with the ones obtained by the WordNet-based Baseline System.

We consider two different scenarios. In scenario $\mathcal{S}_{1}$, we focus on Precision and Recall, we compute the overlap between all tags assigned by each system to each Gold Standard facet, and the actual ground-truth tags in those Gold Standard facets. In scenario $\mathcal{S}_{2}$, we focus on Precision@N.

\subsubsection{Precision and Recall}

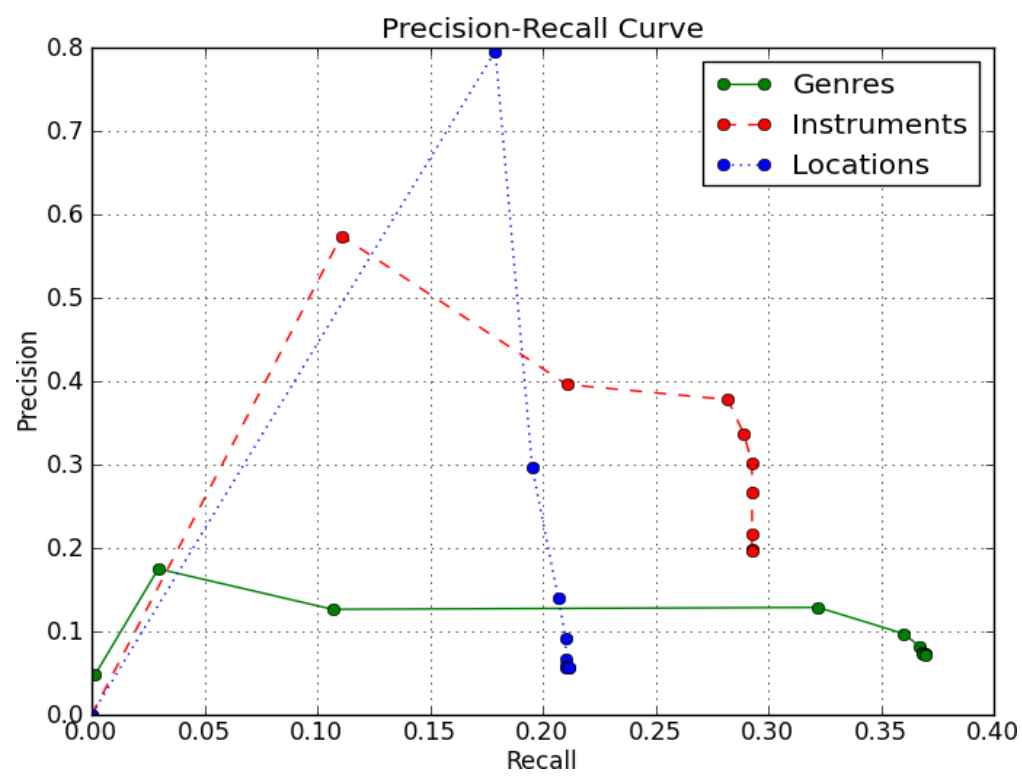

Figure 3: Precision/Recall Curve of the assignment of facets (Genre, Instrument and Location) to tags, when we vary the threshold for attribution of facets to tags in our system. 
Figure 3 depicts the Precision/Recall curve of our system assignments of tags to the facets Music_genres (Genre in the GS), Music_geography (Locale, country, nationality in GS) and Musical_instruments (Instrument in GS). For the Genre and Instrument facets, it seems that threshold of 4 (maxIter in Algorithm 2, i.e. the maximum distance from the tag to the candidate facet) gives the best trade off between precision and recall (largest AUC, or Area Under the Curve), whilst this value is reduced to 2 in the case of Locale, country, nationality. This particular case gives a good insight of our system. In fact, when we impose a threshold of 2, our method is able to assign the facet Music_geography to 140 tags, where $79 \%$ are actually tags in the GS facet Locale, country, nationality. When the threshold is increased to 3, Precision drops dramatically to $29 \%$ (165 out of 559). However, by manually looking at these false positives, one can see that effectively most of them are music genres ("Rai", "Merengue"), artists (due to the nature of the Last.fm tags used in this work ${ }^{11}$ ) or the combination of two facets, such as, e.g., "Spanish_rock" or "Finnish_hip_hop". For instance, given the tag "Rai", which the GS describes as a Genre, our system, based on Algorithm 2 and Equation 1, and using the whole list inferred facets (333), is able to produce the following description:

Rai: \{(Algerian_styles_of_music, 0.67), (Music_genres, 0.16),

(Music_geography, 0.16)\}

which is a more complete semantic description of the tag "Rai". In this case, it seems also clear that a human evaluation (either expert or lay) is needed for evaluating these complex semantics.

Setting our system's threshold to 4 (value chosen after evaluating its performance analyzing the Precision/Recall curve, see figure 3), we compare our system to the Baseline System in terms of Recall (in table 6) and precision (in table 7).

\begin{tabular}{|c|c|c|}
\hline GS Facet (total number of tags) & $\begin{array}{c}\text { Baseline system } \\
\text { classification }\end{array}$ & $\begin{array}{c}\text { Our System } \\
\text { classification }\end{array}$ \\
\hline Genres (711) & $32(\mathrm{R}=0.045)$ & $272(\mathbf{R}=\mathbf{0 . 3 8 2})$ \\
Instruments (280) & $67(\mathrm{R}=0.239)$ & $79(\mathbf{R}=\mathbf{0 . 2 8 2})$ \\
Locations \& Languages (609) & $89(\mathrm{R}=0.146)$ & $133(\mathbf{R}=\mathbf{2 1 8})$ \\
Moods (115) & $3(\mathbf{R}=\mathbf{0 . 0 2 6})$ & $0(\mathrm{R}=0)$ \\
\hline
\end{tabular}

Table 6: Evaluation scenario $\mathcal{S}_{1}$ : Overlap with the Gold Standard ground truth tags in number of tags (Recall value in parenthesis).

An interesting result of table 6 is the fact that our system more than doubles the coverage of Gold Standard tags of the Baseline system: where the Baseline system covers 191 tags out of the 1715 tags of the Gold Standard, we cover 484, this represents an increase of $153 \%$.

\footnotetext{
${ }^{11}$ people tend to tag an artist with the name of the artist
} 


\begin{tabular}{|c|c|c|}
\hline GS Facet & $\begin{array}{c}\text { Baseline system } \\
\text { classification }\end{array}$ & $\begin{array}{c}\text { Our System } \\
\text { classification }\end{array}$ \\
\hline Genres & $103(\mathbf{P}=\mathbf{0 . 3 1 1})$ & $1757(\mathrm{P}=0.155)$ \\
Instruments & $200(\mathrm{P}=0.335)$ & $208(\mathbf{P}=\mathbf{0 . 3 8 0})$ \\
Locations \& Languages & $248(\mathbf{P}=\mathbf{0 . 3 5 8})$ & $1207(\mathrm{P}=0.110)$ \\
Moods & $\mathbf{5 1}(\mathbf{P}=\mathbf{0 . 0 5 8})$ & $0(\mathrm{P}=0)$ \\
\hline
\end{tabular}

Table 7: Evaluation scenario $\mathcal{S}_{1}$ : Number of tags assigned to each facet (Precision value in parenthesis).

On the negative side however, our system is not able to infer tags from the Mood facet. In fact, this facet was not discovered by our method, starting from the seed Music and following our algorithm, our system is unable to reach the facet Mood, although the concept does exist as a category named Emotion in Wikipedia, but our algorithm could not uncover the path in Wikipedia between Music to Emotion.

We can also notice that our system scores worse than the Baseline system in terms of precision (see table 7), except for the case of the Instruments facet. This is due to the fact that our system tends to be much more productive (i.e. it classifies more tags) than the Baseline System. We propose to scrutinize this aspect further by considering an additional evaluation scenario, $\mathcal{S}_{2}$, where the metric is Precision@N.

\subsubsection{Precision@N}

As our system tends to be much more productive than the Baseline system, one could argue that precision values would not be directly comparable because of different numbers of tags retrieved by the two systems. Therefore, the objective of evaluation scenario $\mathcal{S}_{2}$ is to compare precision of the two systems on a comparable base of retrieved tags. For a given facet, we limit the number of tags used in computing precision to the number of tags retrieved by the less productive system, which is always the Baseline system. ${ }^{12}$ For instance, for the Genre facet, we compute the precision with respect to the GS ground truth over a set of 103 tags. In the case of the Baseline system, all tags retrieved are used, while in the case of our system, a selection of 103 tags among the 1784 tags retrieved are used.

In order to select among the tags retrieved by our system those that will be used for computing precision, we retrieve the top-N tags (hence, Precision@N) for each facet (where $N$ is the limit number). For each facet, the tags are ordered by relevance. This relevance is measured as the distance between the facet to the candidate tag (i.e. the lower this value, the more relevant the tag is).

It is interesting to compare the results of table 7 to those of this scenario, described in table 8. Logically, the performance of the Baseline system are the same in both tables, but our system appears to perform better with the Precision@N metrics than with Precision.

\footnotetext{
${ }^{12}$ Except in the case of Mood for which our system does not retrieve any tag (see above). For this reason, we will not consider the Mood facet in evaluation scenario $\mathcal{S}_{2}$.
} 


\begin{tabular}{|c|c||c|}
\hline GS Facet $(\mathrm{N})$ & $\begin{array}{c}\text { Baseline system } \\
\text { classification }\end{array}$ & $\begin{array}{c}\text { Our System } \\
\text { classification }\end{array}$ \\
\hline Genres $(103)$ & $32\left(P_{@ 103}=\mathbf{0 . 3 1 1}\right)$ & $27\left(P_{@ 103}=0.262\right)$ \\
Instruments $(200)$ & $67\left(P_{@ 200}=0.335\right)$ & $76\left(P_{@ 200}=\mathbf{0 . 3 8}\right)$ \\
Locations \& Languages $(248)$ & $89\left(P_{\left.\Theta_{248}=0.358\right)}\right.$ & $118\left(P_{@ 248}=\mathbf{0 . 4 7 6}\right)$ \\
\hline
\end{tabular}

Table 8: Evaluation scenario $\mathcal{S}_{2}$ : Precision@N, where $\mathrm{N}$ varies with the facets.

\begin{tabular}{|c|c|c|}
\hline Aida & Filmi & Political \\
Al_Green & Freak_folk & Polka \\
American_Indian_music & Peorge_Michael & Rautalanka \\
Andreas_Scholl & Gnawa & Religious \\
Art & Gospel_blues & Rhythm_and_blues \\
Bittersweet & Indietronica & Russian_chanson \\
Boy_soprano & Indigenous & Schlager \\
brianmcknight & Indigenous_Australian_music & Selena \\
Cabaret & Islamic & Side_project \\
Cantautori & Jeff_Buckley & Song \\
Chamber & Jesus & Soprano \\
Circus & Levenslied & Sopranos \\
Classical_composers & Lisa_Gerrard & Sufi \\
Contemporary & Maritime & Symphonic_rock \\
Contemporary_Christian_music & Mercedes_Sosa & Tenor \\
Contraltos & Mezzo-soprano & Tenors \\
Countertenor & Mezzo-sopranos & Tin_Pan_Alley \\
Countertenors & Military & Tosca \\
Dansband & Minimalism & Traditional \\
Death & Minimalist & VIA_music \\
Drone & Music_hall & Wedding \\
Electronic_dance_music & Nico & World \\
Ethereal_Wave & Zarzuela \\
Ethnic & Ousrat_Fateh_Ali_Khan & Zolo \\
Featuring & Oldies & \\
Film & Operetta & \\
\hline
\end{tabular}

Table 9: List of the 76 top genres (from the top 103) produced by our system which are not present in the Gold Standard.

One can see in table 8 that our system outperforms the Baseline system on the Instruments and Locations and Languages facets. The Baseline system still shows higher precision on the Genres facet, but in a much smaller measure than in table 7 .

For the case of Genres, it should be noted that relatively low precision does not necessarily mean that retrieved items are bad. For instance, it is informative to consider examples among the 76 (i.e. $103-27=76$ ) top ranked genres that our system retrieves and that are not part of the Gold Standard, as well as the remaining, supposedly not relevant, 71 genres retrieved by the Baseline system that were not part of the Gold Standard either (see Tables 9 and 10 . One can observe that, even if not part of the 


\begin{tabular}{|c|c|c|}
\hline Act & Gregorian_Chant & Postmodernism \\
African-American_Music & Hillbilly_Music & Progressive_Rock \\
Anthem & Hot_Jazz & Prose \\
Aria & Hymn & Psychedelicrock \\
Ballroom & Landscape & Punk_Rock \\
Beat & Lead & Religious \\
Bebop & Light_Opera & Requiem \\
Black & Macumba & Rhythm_and_Blues \\
Blue_Note & March & Rock'n'Roll \\
Boogie & Marching & Scat \\
Cantata & Martial & Scat_Singing \\
Chamber & Mass & Scene \\
Chant & Melodrama & Serious \\
Chorale & Military & Sitcom \\
Christmas_Carol & Modernism & Skiffle \\
Church & Modern_Jazz & Sonata \\
Comedy & Movement & Spiritual \\
Cool_Jazz & Neo_Jazz & Stream_of_Consciousness \\
Country_and_Western & New_Jazz & Symphonic \\
Drama & Operetta & Trad \\
Ethnic & Oratorio & Tragedy \\
Folksong & Personal & Verse \\
Genre & Poetry & Zydeco \\
Gospel & Popular & \\
\hline & & \\
\hline & & \\
\hline
\end{tabular}

Table 10: List of the 71 top genres produced by the Baseline system (from a total of 103) which are not present in the Gold Standard.

Gold Standard, many of the tags retrieved and classified as genres are actually relevant music genres. Both systems were able to retrieve what appears to be very specific genres, such as "Cool_Jazz", "Neo_Jazz" and "Psychedelicrock" (from the Baseline system); or "Zarzuela", "Levenslied", "Schlager", "Rautalanka" (from our system). This illustrates the reported fact (Pachet and Cazaly, 2000) that expert-based taxonomies of musical genres such as the Gold Standard used here tend to be incomplete and outdated, albeit comprising over 700 genres.

Moreover, an advantage of our system over the Baseline is to be geared towards retrieving tags that do not necessarily exist in expert-based taxonomies, while the Baseline is biased to be conservative and to retrieve tags corresponding to expert knowledge (either from the Gold Standard, or from WordNet). Therefore, even if the Baseline appears to have a high precision, it is precisely biased towards high precision, the downside of this being its relatively low recall (much lower than that of our system, see table 6). Further, most of the 71 genres from the Baseline system that are not part of the Gold Standard but that are nevertheless relevant are also inferred by our system, beyond the top 103, e.g. "Zydeco", "Progressiverock", "Punkrock", etc. Finally, looking at examples of genres retrieved by our system also shows tags not present neither in the Gold Standard, nor in the set retrieved by the Baseline, but that are still relevant, such as 
e.g. "Indietronica" or "Ethereal_wave" (in the top 103), and e.g. "Mexican_Cumbia", "UK_Hard_House" (beyond the top 103). The fact that our system can retrieve such genres is interesting especially because they correspond to a very specialized (either culturally or temporally) corner of human knowledge about music, hardly reachable for small groups of experts, but at the reach of folksonomies.

\subsection{Use Case: Assigning semantic facets to artist tags}

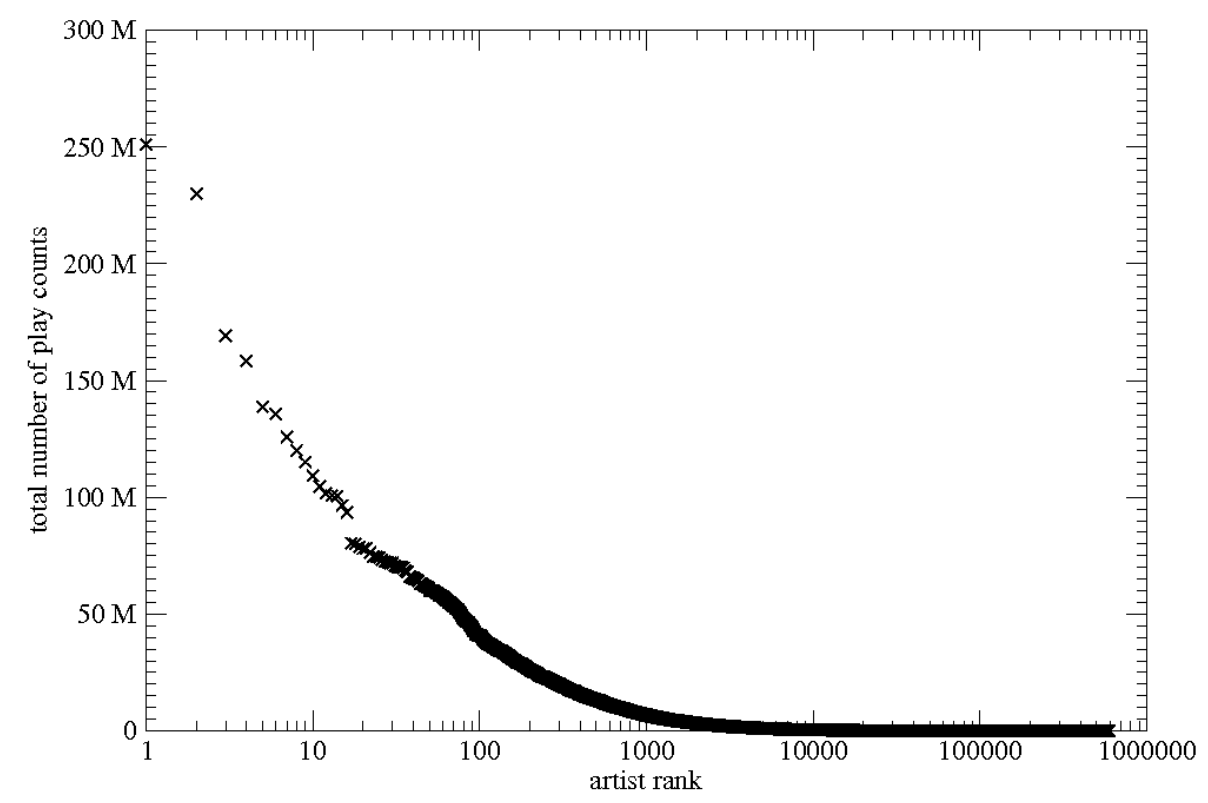

Figure 4: The Long Tail for artist popularity. A log-linear plot depicting the total number of plays. Data gathered during April, 2010, for a list of 594,989 artists.

Here, we wish to verify whether we can produce a meaningful faceted description of music artists' tag sets. We are particularly interested in evaluating the worth of community-based information (i.e. Wikipedia) in generating applicable facets and tag/facet assignments for music artists in the Last.fm community. Furthermore, we focus on artist popularity (number of total playcounts in Last.fm) in order to understand the intrinsic differences that exist in any social tagging system. That is, users tend to tag more the popular artists rather than those that are less known. 


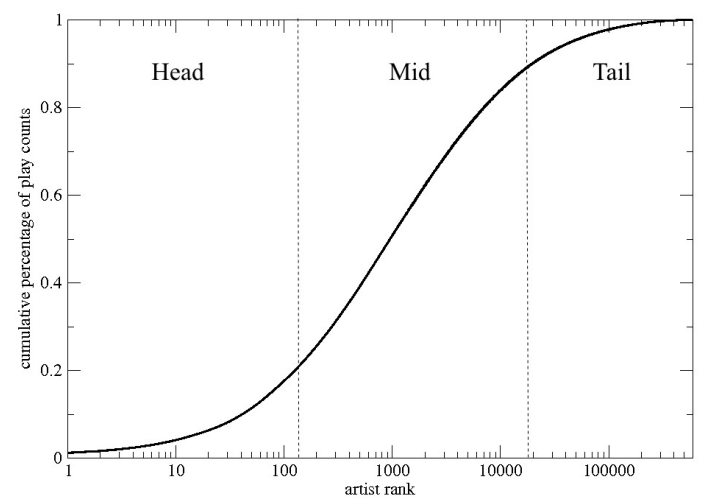

Figure 5: The Long Tail model. It shows the cumulative percentage of playcounts of the 594,989 music artists from figure 4. Only top-955 artists, $0.16 \%$ of all the artists, accumulates the $50 \%$ of total playcounts (N50). Also, the curve is divided in three parts: head, mid and tail $\left(X_{\text {head } \rightarrow \text { mid }}=104\right.$, and $\left.X_{\text {mid } \rightarrow \text { tail }}=10,860\right)$, so each artist is located in one section of the curve.

\subsubsection{Artist Popularity}

Figure 4 depicts the Long Tail popularity, using total playcounts, for 594,989 artists. The horizontal axis contains the list of artists ranked by its total playcounts. E.g. The Beatles, at position 1, have more than 250 million playcounts. The Long Tail model, $F(x)$, simulates any heavy-tailed distribution (Kilkki, 2007). It models the cumulative distribution of the Long Tail data. $F(x)$ represents the share (\%) of total volume covered by objects up to rank $x$ :

$$
F(x)=\frac{\beta}{\left(\frac{N_{50}}{x}\right)^{\alpha}+1}
$$

where $\alpha$ is the factor that defines the $S$-shape of the function, $\beta$ is the total volume share (and also describes the amount of latent demand), and $N_{50}$, the median, is the number of objects that cover half of the total volume, that is $F\left(N_{50}\right)=50$.

Once the Long Tail is modelled using $F(x)$, we can divide the curve (figure 4) in three parts: head, mid, and the tail. The boundary between the head and the mid part of the curve is defined by:

$$
X_{\text {head } \rightarrow \text { mid }}=N_{50}^{2 / 3}
$$

Likewise, the boundary between the mid part and the tail is:

$$
X_{\text {mid } \rightarrow \text { tail }}=N_{50}^{4 / 3} \simeq X_{\text {head } \rightarrow \text { mid }}^{2}
$$


Figure 5 depicts the cumulative distribution of the Long Tail from figure 4.

Now, we randomly select 50 artists in each portion of the Long Tail (head, mid and tail), in order to answer these questions: (1) how many artist tags can we classify?, and (2) how many facets do they correspond to?

In order for the Baseline system and our system to be comparable, we use the same set of 8 facets corresponding to a strict match between inferred and expert-defined facets (see table 5).

\begin{tabular}{|c|c||c|}
\hline Long-tail Portion & $\begin{array}{c}\text { Baseline system assigns } \\
\text { at least one facet }\end{array}$ & $\begin{array}{c}\text { Our system assigns } \\
\text { at least one facet }\end{array}$ \\
\hline Head & $29.4 \%$ & $\mathbf{4 1 . 1 \%}$ \\
Mid & $25.3 \%$ & $\mathbf{3 3 . 4 \%}$ \\
Tail & $16.9 \%$ & $\mathbf{1 7 . 1 \%}$ \\
\hline
\end{tabular}

Table 11: Classify Artist tags (the threshold parameter of our system is set to 4)

\begin{tabular}{|c|c||c|}
\hline Long-tail Portion & Baseline system & Our system \\
\hline Head & 1.74 & $\mathbf{2 . 2 6}$ \\
Mid & 1.58 & $\mathbf{2 . 1 6}$ \\
Tail & 0.84 & $\mathbf{1 . 2 4}$ \\
\hline
\end{tabular}

Table 12: Average number of assigned facets per artist

In order to assign facets to artist tags, we proceed in two steps: (1) for each artist tag, we compute the top facets using Algorithm 2, and (2) we merge the tags' top facets using the following equation:

$$
\Gamma_{a f}=W_{a t} \cdot R_{t f}
$$

where $W_{a t}$ represents the weight ${ }^{13}$ of tag $t$ for artist $a$, and $R_{t f}$ represents the relevance of facet $f$ for the tag $t$ ( $R_{t f}$ is computed using Equation 1). For the Baseline system, since it is biased by definition towards the Golden Standard, we did not consider different relevances for facets. In other words, $R_{t f}=1 \forall(t, f) \in$ Baseline.

Table 11 and 12 present the results. We can observe that, thanks to attributing a higher number of tags per facet, our method assigns on average more facets to artists than the Baseline system (independently of the popularity of that artist). Yet, the more unknown the artist is the less facets and tags we can assign to her.

\subsubsection{An Example}

As an illustrative example, we consider the Elton John example, shown in figure 1. Table 13 shows the process of assigning facets to artist tags described in the previous section. In this example, we fix the threshold parameter of our system to a value of 4 .

\footnotetext{
${ }^{13}$ this weight is given by the Last.fm dataset, and it is computed according to how many times tag $t$ has been applied to artist $a$. The exact formula, though, is a trade secret of Last.fm.
} 


\begin{tabular}{|c||c|c||c|c|}
\hline \multicolumn{1}{|c|}{ Tags } & \multicolumn{2}{|c||}{ Baseline system } & Matched & Our System \\
\hline pop & Matched & Facets & $\mathrm{X}$ & Music_genres, Music_geography \\
\hline \hline classic rock & & Genre & $\mathrm{X}$ & Record_labels \\
\hline singer-songwriter & & & $\mathrm{X}$ & $\begin{array}{c}\text { Occupations_in_music. Music_genres, } \\
\text { Music_performance, Record_labels }\end{array}$ \\
\hline rock & $\mathrm{X}$ & Genre & $\mathrm{X}$ & Music_genres, Music_geography \\
\hline piano & $\mathrm{X}$ & Instrument & $\mathrm{X}$ & Musical_instruments, Aspects_of_music \\
\hline british & $\mathrm{X}$ & Locale, country, nationality & $\mathrm{X}$ & Music_geography \\
\hline $80 \mathrm{~s}$ & $\mathrm{X}$ & Time period, era/epoch & & \\
\hline $70 \mathrm{~s}$ & $\mathrm{X}$ & Time period, era/epoch & & Musicians, Music_people \\
\hline elton john & & & $\mathrm{X}$ & \\
\hline male vocalists & & & & \\
\hline
\end{tabular}

Table 13: Assignment of facets for artist Elton John's tags

Both systems are able to classify correctly the tags "pop", "rock", "piano" and "british". The Baseline system was not able to classify "classic rock" nor "singersongwriter". This is an illustration of the fact that WordNet does not cover a broad description of the Music domain, a similar problem was reported in (Cano et al., 2004) about the Sound Effects domain. On the other hand, our system could classify these tags. The tag "singer-songwriter" is classified as Occupations_in_music, Music_genres and Music_performance, which are correct. However, it also classified it as Record_labels. A broader analysis of our system's resulting network (as described in section 3) permits us to explore the path between this tag and the facet Record_labels (given the threshold limit of 4), namely:

$$
\text { Record_labels } \rightarrow \text { Music_industry } \rightarrow \text { Occupations_in_music } \rightarrow \text { Singer }- \text { songwriter }
$$

Similarly, our system classifies the tag "Classic rock" as Record_labels with the following path:

$$
\text { Record_labels } \rightarrow \text { Music_industry } \rightarrow \text { Radio } \rightarrow \text { Radio_formats } \rightarrow \text { Classic_rock }
$$

After analyzing the Wikipedia categorization, it appears that there are both broaderOf and subjectOf links, in both ways, between Music_industry and Record_labels. While considering that Music_industry is a broaderOf of Record_labels, the opposite does not seem to make sense. This "bug" in the structure of Wikipedia is the reason why an erroneous facet was attributed to these tags. This situation shows that our system is fragile with respect to noise and inconsistencies in the Wikipedia categorization.

Our method was not able to classify the tags "70s" and "80s". This is due to the design characteristics of this system (as in the case of Moods described in section 4.3): our method, starting from the seed "Music", was unable to reach these specific time periods, known as decades, although the facet Decades does exist in fact in Wikipedia. We propose in the next section lines of future work, to remedy both this problem and the problem of fragility to noise in the knowledge base.

\section{Discussion and future work}

When comparing folksonomies to expert-based taxonomies, the former usually have the advantage to be more complete, and more up-to-date. However, the former have the 
drawback of lacking structured categories, i.e. terms are not explicitly related to categories.

In this paper, we focused on the particular domain of knowledge related to Music, and proposed a system addressing the complementary research problems of (1) uncovering the semantic facets of the popular music folksonomy Last.fm, and (2) automatically categorizing music tags according to this set of facets. The Wikipedia repository of knowledge was used as backbone for semi-structured semantic categories. Our system was able to infer 333 semantic facets of music. By comparing these facets to those of taxonomies defined by experts, we showed that our system is able to cover a significant portion of expert descriptions of music. Importantly, we also argued that among the inferred facets, many of those facets that cannot be matched to expert facets are in fact meaningful and do represent valid ways to describe music, at least within the particular realm of the Last.fm folksonomy. We also showed the relevance of our system in the task of tag categorization on a subset of Gold Standard facets (namely Genre, Instruments, and Locations and Languages), and in the task of automatically categorizing tags of music artists. There were however some expert facets of music that our system could not infer, notably Mood. The main reason is that Mood (or Emotion) is a very generic concept, and can be applied in different contexts. It is then not specifically related to music. By exploring only explicitly music-related topics, it did not reach this concept. An additional shortcoming of our system is that it does not specifically address the issue of ambiguous and polysemic tags. If matched in our wikipedia-based music hierarchy, only one of the possible different meanings of a tag will be retained, and a single facet will be attributed to this tag. Another shortcoming of our system is the relatively low coverage of Last.fm tags (after running both processing stages of our proposed system, only 3596 Wikipedia articles could be matched to the initial 39,953 Last.fm tags, i.e. less than 10\%). Although 3596 tags still represent a relatively large vocabulary, compared to typical musical taxonomies, it is still worth considering the reasons for this low coverage. First, our method makes use of relatively simple stringmatching. More sophisticated string-matching techniques would certainly improve the coverage, and would also improve the cleaning of Last.fm tags (here also increasing coverage). Including multi-lingual Wikipedia data would also certainly increase coverage of Last.fm tags, which are not restricted to English.

There are many avenues for future work. First and foremost regarding potential improvements to the proposed system itself. We plan to modify our system so that it includes the advantages of the Baseline system, without its drawbacks. Namely, instead of starting from the single category Music, our system could be based on hierarchies of facets that are known to be relevant to music (through the inclusion of expert-based ontologies), and expand/refine this initial knowledge via specializing the Wikipedia general knowledge base with the same methods as described in this paper. This may permit to combine in the same system both expert knowledge on specific parts of the universe of music with the grounded and updated underlying knowledge of music lovers (Hepp and de Brujin, 2007; Suchanek et al., 2008). For instance, this would certainly alleviate our system's current shortcoming with respect to the lack of description related to Mood, and 
other concepts whose relevance is wider than the sole music realm. It would also solve the problem of facets that were filtered out because of our tag filter, such as "Rhythm" or "Lyrics". It would also improve Recall of our system. Additionally, more information could be gathered from Natural Language analysis of the Wikipedia abstract (or full articles) themselves. In particular, such analysis could potentially improve current assignment of facets to tags (Section 3.4) and the determination of the respective relevance of diverse facets to a given tag (Section 3.5). In particular, once facets and tags are inferred, it would certainly be useful, as a post-processing step, to cross-check their relevance via Natural Language analysis of corresponding Wikipedia articles. This may prove useful in filtering out tags that are irrelevant to a given facet (e.g. "Featuring" in the list of genres in Table 9).

Another interesting extension of the proposed system would be to infer complex hierarchies of concepts instead of the simpler 1-level hierarchy proposed here (i.e. tagfacet). It would be very interesting, for instance, to infer a multiple levels hierarchy of music genres.

Another very relevant avenue for future work is evaluation. We argue in this paper that many facets that cannot be matched to expert facets are in fact meaningful, and a key strength of our approach. In addition to examples of the worth of such novel facets, as those presented herein, systematic procedures should evaluate the quality of these novel facets. We therefore intend to proceed to a manual evaluation of the facets inferred by our system that do not correspond to facets commonly found in expert-based taxonomies. We will proceed to this evaluation via a questionnaire-based methodology targeting different participant profiles: experts of the music business, musicians, experts in the scientific research on social music tagging, and lay music lovers. Experiments described above could also be ran on different datasets, as e.g. Last.fm tags from individual tracks instead of artists, under the assumption that although generally noisier, good track tags may be more accurate. This would require more sophisticated Natural Language Processing techniques than what is described in this paper Other datasets could also be targeted. Further work should also be dedicated to evaluating the worth of the obtained facets and the whole system in a number of tasks, such as music recommendation and browsing, or tag expansion. In particular, we also plan to further study the distributions of music facets with respect to artist popularity and evaluate in what respect our system could be useful for tackling the problem of cold-start in music recommendation (Celma, 2010). Last, but not least, we plan to study different measures for evaluating the relevance of a music facet to a given tag. This would improve the precision of our system in tasks such as tag classification.

In order to ease reproducibility of research and to stimulate further research on the topic, the whole system, its data and source code are available on the website http: //www.dtic.upf.edu/ msordo/wikifacets/.

\section{Acknowledgements}

We would like to thank reviewers of previous versions of this paper for their valuable comments that helped us improve the paper. Fabien Gouyon is supported by the Me- 
dia Arts and Technologies project (MAT), co-financed by the North Portugal Regional Operational Programme (ON.2 O Novo Norte), under the National Strategic Reference Framework (NSRF), through the European Regional Development Fund (ERDF).

\section{References}

Aucouturier, J.-J. (2009). Sounds like teen spirit: Computational insights into the grounding of everyday musical terms. In Minett, J. and Wang, W., editors, Language, Evolution and the Brain, Frontiers in Linguistics Series. Academia Sinica Press.

Bertin-Mahieux, T., Eck, D., Maillet, F., and Lamere, P. (2008). Autotagger: A model for predicting social tags from acoustic features on large music databases. Journal of New Music Research, 37(2):115-135.

Bischoff, K., Firan, C., C., K., Nejdl, W., and Paiu, R. (2009). Automatically identifying tag types. In Proceedings of the fifth International Conference on Advanced Data Mining and Applications, pages 31-42.

Cano, P., Koppenberger, M., Herrera, P., Celma, O., and Tarasov, V. (2004). Sound effect taxonomy management in production environments. In Proceedings of the 25th AES International Conference on Metadata for Audio.

Celma, O. (2010). Music Recommendation and Discovery. The Long Tail, Long Fail, and Long Play in the Digital Music Space. Springer.

Duan, Z., Lu, L., and Zhang, C. (2008). Collective annotation of music from multiple semantic categories. In Proceedings of the International Conference on Music Information Retrieval.

Hepp, M. and de Brujin, J. (2007). Gentax: A generic methodology for deriving owl and rdf-s ontologies from hierarchical classifications, thesauri, and inconsistent taxonomies. In Proceedings of the 4th European Semantic Web Conference, pages 129-144. Lecture Notes in Computer Science, Springer Verlag.

Kilkki, K. (2007). A practical model for analyzing long tails. First Monday, 12(5).

Lamere, P. (2008). Social tagging and Music Information Retrieval. Journal of New Music Research, 37(2):101-114.

Laurier, C., Sordo, M., Serrà, J., and Herrera, P. (2009). Music mood representations from social tags. In Proceedings of the International Conference on Music Information Retrieval.

Levy, M. and Sandler, M. (2008). Learning latent semantic models for music from social tags. Journal of New Music Research, 37(2):137-150. 
Overell, S., Sigurbjörnsson, B., and van Zwol, R. (2009). Classifying tags using open content resources. In Proceedings of the Second ACM International Conference on Web Search and Data Mining, pages 64-73.

Pachet, F. and Cazaly, D. (2000). A taxonomy of musical genres. In Proceedings of the Content-Based Multimedia Information Access Conference.

Pachet, F. and Roy, P. (2009). Improving multi-label analysis of music titles: A large scale validation of the correction approach. IEEE Transactions on Audio Speech and Language Processing, 2(17):335-343.

Page, L., Brin, S., Motwani, R., and Winograd, T. (1999). The PageRank citation ranking: Bringing order to the web. Technical report, Stanford InfoLab.

Raimond, Y., Abdallah, S. A., Sandler, M. B., and Giasson, F. (2007). The music ontology. In Proceedings of the International Symposium on Music Information Retrieval, pages $417-422$.

Raimond, Y., Giasson, F., Jacobson, K., Fazekas, G., Gangler, T., and Reinhardt, S. (2010). Music Ontology Specification. http://musicontology.com/. Last accessed Jul. 2013.

Sarmento, L., Gouyon, F., and Oliveira, E. (2009). Music artist tag propagation with wikipedia abstracts. In Proceedings of the Workshop on Information Retrieval over Social Networks, European Conference on Information Retrieval.

Sigurbjörnsson, B. and van Zwol, R. (2008). Flickr tag recommendation based on collective knowledge. In Proceeding of the 17th international conference on World Wide $W e b$, pages 327-336. ACM.

Suchanek, F. M., Kasneci, G., and Weikum, G. (2008). YAGO: A large ontology from wikipedia and wordnet. Journal of Web Semantics, 6(3):203-217.

Turnbull, D., Barrington, L., Torres, D., and Lanckriet, G. (2008). Semantic annotation and retrieval of music and sound effects. IEEE Transactions on Audio, Speech and Language Processing, 2(16):467-476.

Westergren, T. (2010). List of Music Genome Project attributes. http://en.wikipedia. org/wiki/List_of_Music_Genome_Project_attributes. Last accessed Dec. 2012, now defunct. 